\title{
Automated Guided Vehicle Systems, State-Of-The-Art Control Algorithms and Techniques
}

\author{
Matthias De Ryck*, Mark Versteyhe ${ }^{\dagger}$ \\ Faculty of Engineering Technology, KU Leuven \\ Spoorwegstraat 12, 8200 Bruges, Belgium \\ Email: *matthias.deryck@kuleuven.be, ${ }^{\dagger}$ mark.versteyhe@kuleuven.be
}

\begin{abstract}
Automated Guided Vehicles (AGVs) form a large and important part of the logistic transport systems in today's industry. They are used on a large scale, especially in Europe, for over a decade. Current employed AGV systems and current systems offered by global manufacturers almost all operate in a centralized way. One central controller controls the whole fleet of AGVs. The authors do see a trend towards decentralized systems where AGVs make individual decisions favoring flexibility, robustness, and scalability of transportation. With the paradigm shift of Industry 4.0 and future requirements, more research is done towards the decentralization of AGV-systems. And global leading manufacturers start to take an active interest. That said, this implementation seems still in infancy. Currently, literature is full of research on central as well as on decentral control techniques and algorithms. For researchers in the field and for AGV developers, it is hard to find structure in the growing amount of algorithms for various types of applications. This paper is, to this purpose, meant to provide a good overview of all AGV-related control algorithms and techniques. Not only those that were used in the early stages of AGVs, but also the algorithms and techniques used in the most recent AGV-systems, as well as the algorithms and techniques with high potential.
\end{abstract}

AGV-systems, State-Of-The-Art, Control Techniques, Centralized, Decentralized, Potential Future techniques

\section{INTRODUCTION}

AGVs were conceived in 1954 [1] by Barrett Electronics Corporation, Illinois, a manufacturing company in the United States. Since then AGVs became widespread all over the globe in every logistics environment where goods are transported. Currently, the market of AGVs is growing fast and is very dynamic. A market report published by Grand View Research (2017) [2] has made a forecast for the period from 2018 to 2025 focusing on the potential growth opportunities of AGVs. Despite AGVs were invented in the United States, this report states that the biggest market of AGVs is now situated in Europe. This will also continue in the future. In the report, they state that the future growth of AGV systems is caused by the emergence of flexible manufacturing systems, the rising demand for customized AGVs and the adoption of industrial automation by SMEs. The growth rate of AGVs in this forecast period will be the highest in Europe, especially in e-commerce and material handling. The current AGV-systems are well known and widely implemented in manufacturing, medicine, and logistics. In these systems, a fleet of AGVs is organized in a centralized way. Tasks like path planning and allocation of tasks are done by a central entity for all the AGVs together. But driven by future requirements like flexibility, robustness, and scalability, the current trends in AGV systems are customization and decentralization. The market report also states decentralization as one of the future technologies which will gain great attention. Other emerging technologies are dual-mode (hybrid)-AGVs which can operate both manually and automatically, camera vision, Light Detection and Ranging (LiDAR) for navigation, and collision avoidance. The most important reasons why this shift is necessary is the enlargement of the AGV fleet. In future systems, larger and more complex systems will be needed to fulfill the transportation demand within a factory. This will not be feasible with currently employed systems because of memory, communication and computation limits. A lot of universities and companies are, for this reason, currently doing research towards decentralization. Although central systems are still prevalent, a paradigm shift towards more decentralized systems is real and necessary. Over the years, an enormous amount of literature has been accumulated in the research on AGVs. And certainly with this paradigm switch towards decentralization. There are already review papers on AGV systems [3], [4] which try to combine this rich amount of literature. However, these are no longer up to date. Because of the new trend towards decentralization, a more up to date review is needed which compares the older well-known centralized techniques and the newer decentralized approaches. For this reason, this paper makes an overview of the current status of control algorithms and techniques for AGV systems. Not only for current employed centralized systems but also for future technologies and potential decentralized algorithms. We will cover every core AGV task needed to control an AGV fleet and we will give our opinion related to potential future techniques. The remaining chapters are organized as follows. In section III. the core tasks needed to let a whole AGV-system work are described. In the following sections III until [VII every core task is deepened out mentioning the current existing stateof-the-art algorithms and techniques. To end each of these sections, a conclusion is made regarding which algorithms or techniques will be more prevalent in the future. Finally, we draw conclusions in Section VIII 


\section{CORE AGV TASKS}

A complete AGV-system consists of multiple core tasks to be able to operate in complex environments. If we take an e-commerce warehouse as a running example then these core tasks can be easily labeled. In such an e-commerce situation, there is a continuous stream of orders which enters the logistical warehouse system $\left(\mathrm{ERF}^{1}\right.$ or $\mathrm{WMS}^{2}$ ). An order can be seen as an object somewhere in the warehouse that needs to be brought to the picking station where the order can be sent to the customer. In this paper, the authors divide the total AGV-system in the following five core AGV-tasks:

- Task Allocation

- Navigation

- Path Planning

- Motion Planning

- Vehicle Management

A first core AGV task is Task Allocation. A set of tasks (orders) which has to be distributed to the fleet of AGVs need to be allocated to a specific AGV in an optimal way. The easiest way to solve this is to allocate the task to the $\mathrm{AGV}$ which is closest to the position of the ordered object. Once a task is allocated, a next core AGV task is used to find the shortest path to the destination. This task is named Path Planning. It uses a representation of the environment to search for a sequence of segments to reach the goal as fast as possible. For Path Planning, it is important that the AGV can navigate properly in its environment. Thus Navigation is also an important core AGV task. If a Path Planning algorithm computes the shortest path for an AGV, this does not mean that the AGV is able to follow that path without any problems. An unforeseen object or person can block the path or other AGVs may need some segments of the path at the same time. To avoid collisions or situations where multiple AGVs enter a life- or deadlock situation, there is another core AGV task named Motion Planning. This planner tries to avoid collisions with other static or dynamic objects. It tries to avoid deadlock situations and tries to limit the number of vehicles in a particular area. Limiting the numbers of vehicles in an area is called zone control. Once the collision-free path is executed and the AGV reaches its destination, the object can be loaded on the AGV. The exact same tasks are then used to bring the loaded object to the picking station. Parallel with all these core AGV tasks, there is another core task, vehicle management, which controls and monitors the status of an AGV. Some management issues are battery lifetime, maintenance requirements, and error status handling.

In the following sections, every core AGV task will be treated and relevant algorithms and techniques will be described.

\section{TASK ALLOCATION}

Task allocation is one of the most challenging AGV tasks. It has as a purpose to assign a set of tasks to a set of robots. This is a constrained optimization problem in which we want the cost of the total assignment to be as low as possible. The

\footnotetext{
${ }^{1}$ Enterprise Resource Planning
}

${ }^{2}$ Warehouse Management System optimization of this problem is NP-hard [5], which means that if we have a lot of tasks and robots, the number of solutions will be enormous. So there does not exist an efficient algorithm which can produce an exact solution to the problem in a finite amount of time. To solve these NP-hard problems, we use approximate optimization techniques named heuristics [6] and meta-heuristics [7]. A task in task allocation can be an object that has to be picked up at a certain location and has to be dropped off at another location in a factory. Another task can be a surveillance task in which a group of AGVs must scan an area in the most efficient way. In the following sections, some properties and solution methods are described.

\section{A. Desired properties}

In task allocation for AGV-systems, there are some desired properties [8] to be considered. One of these properties is divisibility. It is important that the total work is divided in an efficient way. The purpose is to have a high usage of every resource. We don't want busy AGVs on one side and idle AGVs on the other at the same time. Another important desired property is fault tolerance. The task allocation must operate correct no matter what failures are faced.

To meet future system requirements, some additional important properties are scalability, flexibility, and responsiveness.

- Scalability is the way the system can be enlarged without problems. The task allocation algorithms must keep working with a bigger amount of AGVs without reaching memory or computation limits.

- Flexibility means that the algorithm should, in any case, continue operating by continuously adapting to changes in the system.

- Task allocation should also be responsive, this means that it must have high performance also in dynamic environments.

To take all these desired properties into account, proper algorithms are developed. Section III-E covers these algorithms.

\section{B. Taxonomy of tasks}

Every application requires another kind of task allocation. Gerkey and Mataric [9] have proposed a widely accepted taxonomy for task allocation in multi-robot systems. They divide the tasks into the following categories:

- Single robot tasks (SR): Tasks which only need one robot to be completed.

- Multiple robot tasks (MR): Task which needs more than one robot to be completed.

- Single task robots (ST): Robot which can only perform one single task at a time.

- Multi-task robots (MT): Robots which can perform more tasks simultaneously.

- Instantaneous assignment (IA): Tasks are independent of each other and there is no planning for future allocations. The available information about the task only permits an instantaneous allocation.

- Time-Extended assignment (TA): Tasks are dependent on each other. Future allocations can be planned considering 
several constraints. See section III-C for some dependency constraints.

A task allocation situation can be described by a triplet of these categories. The most simple situation is the triplet: SR-ST-IA In which simple independent tasks, only requiring a single robot, are executed by robots which can only perform one task at a time. An extra division in task allocation problems can be made between static and dynamic allocation.

- Static task allocation: The tasks which are allocated are completed by the robot to which the task was initially allocated. Tasks cannot be re-assigned.

- Dynamic task allocation: The tasks can be re-assigned if there is another robot which is better to suit the task then the robot which was initially assigned to the task.

Many types of tasks can occur. For this reason, a bunch of algorithms is developed in past decades to meet all these different kinds of tasks. Section III-E covers these algorithms.

\section{Task Constraints}

In some applications, tasks are independent of each other and are assigned to a robot from the moment the task is available. The only relevant information about the task is a starting and an ending point. After assignment, the robot can directly execute the task knowing this information. This can be the case in a warehouse environment where an order enters and the task for the AGV is to get the ordered object and bring it to the picking station. Knowing the basic information, the task can be assigned to the robot which is closest to the task This is the simplest case. But in real-world applications, we are dealing with several constraints [8]:

- Tasks can have a time window in which tasks can have a duration, minimum starting time, and maximum ending time (deadline). These are time constraints related to the specific task and are called temporal constraints.

- There are also constraints which cause that tasks are dependent on each other. These are called precedence constraints. Tasks can be partial ordered, which means that some tasks must be completed before or after another task. Tasks can be coupled, which means that two or more tasks must be executed at the same time. There can also be incompatibility, in which tasks produce or obsolete other tasks.

- Some further kind of constraints which can restrict some assignments are mobility interferences. For instance when a narrow aisle exists where only one robot can pass on the way to the task.

- A last type of constraints is resource constraints. These can prevent a task to be executed when resources are empty. The AGV then has to be charged before it can execute more tasks.

For the expression of all these types of constraints, representations like Simple Temporal Networks (STNs) [10] or Hierarchical Task Networks (HTNs) [11] can be used. The presence of this variety of constraints has as a result that task allocation in multi-robot systems can get quite complex.

\section{Optimization Objectives}

In the allocation of robots to a set of tasks, there is always a certain objective to be optimized [8]. There are several optimization objectives which can be used.

At first, there are several elements which can be optimized:

- Cost: Cost that it takes for a robot to execute a task. This can be travel cost like time, distance, or fuel consumption.

- Fitness: How well an agent can perform a task.

- Reward: Gain of completing a task.

- Priority: Urgency of completing a task.

- Utility: The subtraction of cost from reward or fitness.

Knowing these elements, there are some types of objectives possible:

- MinMax: Minimize the cost of the worst agent.

- Egalitarian: Maximize the utility of the worst agent.

- TotalSum: Minimize the sum of individual costs.

- Maximize the sum of individual utilities.

- Minimize the average cost per task.

- Maximize throughput.

Different solution approaches use different optimization objectives to get to the global optimum. These solution approaches will be discussed in the next section.

\section{E. Solution Models}

In the last decade, there has been done a lot of research to solve the problem of multi-robot task allocation. It is a vast area because of the huge diversity of tasks and task constraints. A lot of solution approaches have been developed and are available in the literature. This makes the structuring of task allocation algorithms rather difficult. In this review paper, the authors want to make two clear separations in the state-of-the-art solution algorithms in an attempt to structure the huge amount of algorithms. In the way the algorithms are structured, we made a distinction between centralized and decentralized structures. And in the way the problem is solved, we made a distinction between Optimization-based solutions, Market-based solutions, Behavior-based solutions, and Field-based solutions. Each of these four mentioned solution methods can have both a central or a decentral structure.

1) Subdivision regard to the algorithm structure: The nature of task allocation algorithms can be roughly divided into centralized and decentralized approaches. And this in the manner that they are organized. This means that information, solution algorithms or intelligence in general, can either be collected in a central unit or can be distributed over the total system.

a) Centralized approaches: In centralized approaches [12], there is one single control unit which has all the information available of the whole system to allocate the tasks. This is information like the individual positions of AGVs, tasks with their time window or other constraints, or costs for the AGVs to get to each task in the form of travel cost (time, distance, or fuel consumption). Using the full 
information set, the centralized unit can use a search algorithm to search in the total solution space for the best possible solution which meets the global optimum. Centralized approaches mostly have very good quality assignments for small groups of AGVs and static environments because they have access to global information. They are easy to implement but lack robustness as the central computer acts as a single point of failure. Also, they lack scalability because the more AGVs are operating, the more information the algorithm has to consider and the more communication bandwidth, memory, and computing power needs to be available. Centralized approaches are widely used because they can produce solutions which are in the global optimum. Although in practice, it is known that with the time, when system parameters are changed, this is not the case anymore. To conclude: Centralized approaches are well known and widespread in the industry, but do not meet future requirements like openness, flexibility, scalability, and robustness.

b) Decentralized approaches: In decentralized approaches [12], the total intelligence is distributed over the system components instead of being collected on one computer. In this approach, solution algorithms have access only to local information. An AGV which uses this decentralized structure can make decisions/optimization's regard to its own information which it has from itself and from neighboring AGVs. This makes it a stand-alone and self-thinking device which tries to make the best decisions for its own. As these algorithms do not have global information, it is harder to come to a globally optimal solution. As a consequence, these approaches result in sub-optimal solutions. However, they are much more flexible and robust than the centralized algorithms. Decentralized approaches give good results in dynamic environments with a large group of AGVs operating. Despite it is difficult to implement, this system is more robust and scalable. Decentralized structures gain high attention in the current $\mathrm{AGV}$ market but are not yet well-known and widespread in practice.

c) Hybrid approaches: As nothing in the world is either black or white, there are also hybrid approaches in which the task allocation can have both central as well as decentral properties. AGVs can, for instance, use their local information to compute certain costs to execute the tasks. But where the costs of all AGVs is evaluated by a centralized algorithm. In practice, every system is a hybrid system. But currently, most of the systems are more central then decentral.

2) Subdivision regard to the solution method: The way of solving task allocation algorithms can be divided into optimization-based, market-based, behavior-based, and fieldbased solutions. These are covered in the next sections.

a) Optimization-based solutions: In optimization-based solutions [12], an algorithm searches for an optimal solution in a solution space which maximizes a profit or minimizes a cost using all the information it has access to.

If the solution space is small, exact solutions can be used. For a small number of tasks and robots, it is, for example, feasible to keep up a matrix with traveling costs for each robot to get to each task. Taking the constraints into account, it is then possible to use an exact algorithm which can find the optimal solution of task assignment in this small solution space in a finite amount of time.

If the amount of tasks and robots increases significantly, the solution space becomes too large. In this situation, no exact solution methods can be used. These problems are called NPhard and no exact algorithms exist which can find the optimal solution in a finite amount of time. In this case, approximate search algorithms need to be used. These are called heuristics and meta-heuristics. They are used to find an approximate solution in the large solution space which leans the best to the global optimal solution. Also, algorithms which can search for the best heuristic to use for a particular problem exists. These are hyper-heuristics.

- Exact algorithms: A first exact searching algorithm is Mixed-Integer Linear Programming (MILP) [13], where the objective functions and constraints are formulated in integer and linear equations and solved by a certain solver program. Another exact algorithm is Brute Force Search or Exhaustive Search [14]. This algorithm evaluates every possible solution and selects the best. The Branch and Bound algorithm [15] is also an exact algorithm. In this algorithm, the set of possible solutions is represented as a tree with the whole set of solutions at the root. The algorithm explores branches of this tree which represents subsets of solutions. For each branch, an upper and lower bound is defined. The algorithm enumerates candidate solutions of a branch if this branch can produce better solutions then the solution already found. Otherwise, the branch is discarded and no solutions in this branch will be evaluated. Also other tree-based algorithms [16], [17] can be used to find an exact solution.

- Heuristics: Heuristics like Random Search [18], NSA [19], or Hill-Climbing [20] take a solution every time step during a fixed amount of steps and compare it to the best solution already found. The best solution yet found after the amount of steps to execute is considered as the best solution.

- Meta-heuristics: Heuristics only look for better solutions when comparing. Using this technique, they can get stuck in local optima. Therefore, meta-heuristics are used which also temporarily allow worse solutions at some times to get out of the local minimum. These are actually strategies to guide a search process. Meta-heuristics can be divided into trajectory-based and population-based methods:

- Trajectory-based methods are methods where a solution space is searched and where the probability of choosing a better solution above a worse one is dependent on the moment of the trajectory in the timespan of the algorithm. Examples are Simulated 
Annealing [18], [21], Iterative Local Search [22], Variable Neighborhood Search [23], and Tabu Search [18], [24].

- Population-based methods are methods where populations are used to search the solution space. Examples are Genetic Algorithms [25], [26], Particle Swarm Optimization [27], Memetic Algorithms [28], and Ant Colony Optimization [29].

- Hyper-heuristics: Hyper-heuristics [30] are used to automate the process of selecting, combining, adapting, or generating several heuristics to solve search problems. There are a lot of heuristics which can be chosen to solve a problem. Each has their own weaknesses and strengths. Hyper-heuristics try to automatically choose proper heuristics out of a set of low-level heuristics at any given time dependent on the current state of the problem.

- Dynamic Programming: Dynamic programming [31] is another method to solve optimization problems which do not really fit into the above-mentioned structure. It is actually a method to solve problems in general. In dynamic programming, the total problem is divided into sub-problems of which a solution can be found in a more simple way than the solution of the total problem. This is the case if the sub-problems can be recursively nested into the global problem. The total complex problem can then be solved by using the solutions of the simpler subproblems. Results of sub-problems are stored in a kind of table. When solving the total problem, these solutions can be used when needed. In optimization, dynamic programming simplifies a decision by breaking it down into simpler decisions.

Next to the mentioned popular solution algorithms, there also exist a lot of variants on them to meet all the different types of tasks and task constraints. A mix of heuristics can be used for instance in certain applications. This is called "hybrid heuristics" [24]. Heuristics and meta-heuristics are frequently used for searching a near-optimal solution in large-scale optimization problems. These optimization algorithms are very extended and deeply worked out. Our interest is more focused on the market-based solutions covered in Section III-E2b, which will be deepened out more. For this reason, the taxonomy of optimization-based algorithms was only sketched.

b) Market-based solutions: In market-based solutions [12], an economic principle is used to solve the task allocation problem. In this case, the allocation is not done by executing an optimization process using all available information. But a specific method of auctions is used where each robot uses its local information to calculate bids. The principle of an auction is easy to understand, and the most basic approach is the CNET protocol [32]. In this protocol, an auctioneer announces one single task at a time to bidders. Each bidder places a bid for the task dependent on the cost for the bidder to execute the task. The auctioneer then evaluates all the bids, including its own bid, and assigns the task to the robot with the highest bid. This is called a greedy solution as the simplest best solution is chosen. After a robot is assigned to a task, it can no longer bid on another task. This is the simplest marketbased approach but there are a lot of variants to this:

- First of all, the role of the auctioneer can be played by a central computer, by a fixed robot, or can be altered between robots based on the winner, at random or via token passing. This causes that a market-based approach can be central or decentralized from structure.

- Second, the way of offering tasks to the robots can be different. There can be one single item auctioned at a time. This is called a sequential single-item auction and is used in most of the algorithms like CNET [32], SIT-MASR [33], CBAA [34], and OCA_Alloc [35]. Also, a bundle of items can be auctioned together. This is called a combined auction. In this last category, there are another two options. Robots can bid on a bundle of tasks but with one bid for each task. This is called parallel single-item auction. The task which received the highest bid is then allocated to the robot which offered this highest bid and the unallocated tasks are auctioned again. This is applied in the $\mathrm{M}+[36]$ and Prim Allocation [37] algorithm. The robot can also bid on a combination of tasks in which it has one bid for a cluster of tasks. This last one is called combinatorial auction and is used to prevent synergies. The CBBA [34] and SET-MASR [33] algorithms use this approach.

Synergy: If there are two tasks and the cost for one robot to go to each task separately is higher then if it does the tasks in sequence (combination), then this is called a positive synergy between these tasks. This is the situation when both tasks are located near each other. A negative synergy between tasks means that the tasks are located far from each other and the cost for the robot to visit each task separately is smaller than to visit the tasks in sequence.

Combinatorial auctions are known to have solutions near to the global optimum. But this has as a price that they are computationally heavy because of the number of possible task combinations which is exponential in the number of tasks. Sequential single-item auctions are mostly used because they are simple yet effective. A comparison between these kinds of task offering approaches can be found in [38].

- Further, a rule of participation can alter. There can be a method where a robot which won a bid is not allowed to the auction anymore. Or it can be possible that no matter how many tasks a robot is already assigned to, it can still bid on tasks and participate in the auction.

- Also the way bids are calculated can be different. Bids can be calculated using the cost for the robot to perform 
a task in the simplest case. This simple bidding is used in CNET, OCA_Alloc [35], CBAA [34], and CBBA [34]. But in the Prim Allocation [37], SIT- and SET-MASR algorithms [33] for example, the bids are calculated using the marginal cost to execute a task.

Marginal cost: If there are two tasks of which task one lays on a distance of $5 \mathrm{~m}$ from the robot and task two on a distance of $10 \mathrm{~m}$ from the robot and on a distance of $3 \mathrm{~m}$ from task one. The total cost for the robot to execute each task separate is $15 \mathrm{~m}(5+10)$. But if the robot is already assigned to task one (cost $5 \mathrm{~m}$ ), then after executing this task, it only takes the extra cost of $3 \mathrm{~m}$ to execute task 2 . This extra cost is the marginal cost which is also used to prevent synergies. In this situation, there is a positive synergy between the two tasks.

- Market-based approaches also have to deal with constraints. There exist variations on the standard mentioned algorithms which take these constraints into account. A first variation on the sequential single-item auction algorithm is the TeSSI algorithm [39]. A simple single-item auction where robots can bid on more tasks at the same time is used. But while bidding, each robot takes time constraints into account using a Simple Temporal Network (STN). This network is an individual schedule for each robot which uses it to find a free place in this schedule for a new task before it bids on it. Another variant on the sequential single-item approach takes precedence constraints into account [40]. And another combines the previous two and considers both temporal and precedence constraints [41]. As a last variant on the sequential single-item approach, a variant considering resource constraints is proposed in [42].

- Some market-based algorithms also add an extra consensus phase to the auction process to better the quality of the assignments. This quality can be poor when only using auctions as a task allocation algorithm. A consensus in this sense is a further transfer of tasks between robots after the tasks are assigned by the auction. So there is a constant re-assignment of tasks during the operation of the AGVs. The above mentioned SIT-MASR [33] algorithm also uses consensus by exchanging single tasks after the auction. A variant on this algorithm is called SET-MASR [33]. In this algorithm, the negotiation is done with a set of subtasks instead of single tasks to improve the quality of solutions. An article which proves the advantages and increase in solution quality of task switching is [43]. Algorithms which also use consensus are CBAA, CBBA [34] and OCA_Alloc [35].

- The last variant is the S+T algorithm [44]. Also an auction-based algorithm is used but tasks which need more than one robot are included. So if the robot reaches a task which it cannot handle alone, it is going to start another auction to receive services (from other robots). The cost of the task will then be the added cost of the robot and the service.

To conclude, there exist a lot of variations to the simple auction principle. Other market-based approaches are TraderBots [45], MURDOCH [46], and DynCNET [47]. In general, market-based approaches are robust and scalable. The optimization is caused in a selfish way as every AGV looks at its own resources and calculates a bid on this. But if every AGV tries to optimize itself, then also the global situation will be optimized. Another important advantage is that this way of allocating is very traceable and understandable. This in contrast to optimization algorithms where complex searches are done which are not traceable at all. This makes it difficult to monitor the task allocation process. These market-based approaches are for these reasons good candidates to do task allocation in future decentralized systems.

c) Behavior-based solutions: In behavior-based solutions, robots use motivational behaviors such as impatience and acquiescence. Using these behaviors, robots can motivate the ability to perform a task or they can give up tasks they are not able to perform. Algorithms for this type of solutions are Alliance [48], Ble [49], and Vacancy Chains [50]. As these algorithms are not very popular in task allocation, we will not go further into them.

d) Field-based solutions: A very specific type of solution is the field-based solution [51]. This one is not frequently used but can be added to another solution method to improve solutions. The essence of the algorithm is that a robot moves along a potential field which consists of attracting fields emitted by the goal and repelling fields which are emitted by obstacles and other AGVs. The supper position of those fields generates a total field of which the robot follows the gradient until it reaches the goal.

\section{F. Conclusion}

In this section, we went through a lot of different task allocation algorithms with different characteristics and solution methods. A lot of these algorithms are studied well and are implemented in practice. But because of the huge amount of algorithms, it is difficult to find the appropriate method for each application. Nowadays, a lot of centralized approaches are implemented which make use of optimization-based algorithms which uses all possible information to make an optimal solution. But in the future, as many manufacturers may want to decentralize their systems and introduce flexibility and scalability, more market-based solutions will be used. These already have a decentralized character and they are easy to understand and to monitor. In the opinion of the author, more sequential single-item auction-based algorithms which introduce consensus to have better quality assignments will be used. This is a combination of all the advantages of other algorithms without being too complex. As an addition, these algorithms will take into account several types of constraints, and will allow each AGV to bid on more tasks using marginal cost bids. 


\section{NAVIGATION}

For a vehicle to move inside any area, it is obvious that navigation is crucial thus certainly also in AGV-systems. In contrast to the other tasks, it makes sense that this task is already decentralized. All the navigation equipment and software are on board. Each AGV needs to navigate itself inside the environment. Information about the location in the 2D-map of the environment can then be sent to a central computer or to the other devices. This will be a 3-degrees of freedom coordinate set which consists of the $X$ - and $Y$-coordinates and a rotation angle. In the past decades, there is also been a lot of improvement in navigation systems [52], [53]. There are some old school techniques which are still used. But there are some newer techniques which will gain more attention in the future.

Some of the older and proven techniques are:

- Inductive navigation

- Optical navigation

- Magnetic navigation

- Inertial navigation

A well known and standard in current AGV-systems is the Laser navigation which makes use of the triangulation theory. Some new techniques which are rapidly gaining attention are:

- GPS navigation

- Natural navigation

- Vision guided navigation

The next part of the paper will go through some common types of navigation. In current AGV systems, the AGVs move on a predefined circuit of paths. In academia, sometimes an AGV can move freely into an area without being fixed to a circuit but this is almost not used in the industry. Thus in current industrial systems, the navigation methods are used to stay on this path. The circuit can be physically present on the factory floor in the form of a tape or a wire or can be virtually present in the internal map of the AGV designed by graphical design software. It is clear that all the old navigation types are navigation methods which use a physical circuit. For future requirements like flexibility, it is not efficient to have fixed physical paths which are difficult to adapt. Virtual navigation is much more flexible as a change in the circuit can be done easily online in the graphical design software. The navigation methods can be divided into physical and virtual path navigation.

\section{A. Physical path navigation}

With physical path navigation, the paths are present as physical guidelines on the floor and the navigation is easy as there is only a sensor needed to detect the guideline and make that it is followed. The predefined paths are fixed on the ground using tape or embedded into the ground using a wire. So the AGV does not actually know its position inside the area map but just stays on the guideline. Marks along the guideline tell the AGV if it has to take a special action like increasing or decreasing speed, or to rotate at a certain degree when being in a curve.

1) Inductive navigation: This is the first type of navigation used in the first generation of AGVs [1], [54]. In this type of navigation, a wire is embedded into the floor. There is a current that runs through the wire which maintains a magnetic flux. The AGV has a sensor on board which consists of coils which can detect the emitted magnetic flux. The sensor detects deviation from the wire due to a decrease in magnetic flux and sends this to a controller which adapts the speeds of the wheels to go back on track. This is a very accurate method but the wired paths are fixed into the ground and cannot be adapted easily without any damage. However, this technique can still be used in very small aisles to perfectly stay on track.

2) Optical navigation: In this type of navigation, a color tape or a painted line with high contrast with the ground color is placed onto the floor [55]. The AGV has an optical sensor on board which can detect the tape. The sensor detects deviation from the tape and sends this to a controller which adapts the speeds of the wheels to go back on track. As these paths are physically present onto the floor, it is not very flexible to make changes to the circuit. A tape has to be removed and replaced when the circuit has to be changed. Another disadvantage is that the tape can become dirty or can be damaged. Yet it is easier to adapt then the above wire method. This option is also cheap because of the tape and the only use of an optical sensor.

3) Magnetic tape navigation: The physical guide path is marked with magnetic tape that is placed onto the factory floor [56]. Inside the vehicle, there is a magnetic sensor that can detect the magnetic field. The sensor detects deviation from the tape due to a decrease in the magnetic field and sends this to a controller which adapts the speeds of the wheels to go back on track. It is not very flexible to make changes to the circuit, but still more flexible than the wire option. A tape has to be removed and replaced when the circuit has to be changed. The disadvantage in comparison to a wire is that the tape can become dirty or can be damaged. The advantage of this option is that it is cheap because of the tape and the only use of a magnetic sensor.

\section{B. Virtual path navigation}

With virtual path navigation, the paths are virtually present inside the local map maintained by the AGV or in the global map of the central unit. This makes it easily adaptable and expandable online. Navigation is more difficult because the AGV needs to know its exact position into the virtual map and needs to calculate deviations from the path in the software.

1) Magnetic spot navigation: A grid or line of spots is embedded into the floor [57] on specific $(x, y)$-coordinates in the area map. The spots can be passive permanent magnets or transponders. Inside the vehicle, there is a magnetic sensor that can detect the spots. By detecting the spots, the AGV can 
determine its absolute position in the map. Together with an encoder on the wheels which calculates the traveled distance (odometry), this can be very accurate. A disadvantage is that this method is time expensive to install and to modify.

2) Laser navigation: Laser navigation [58] is currently the most popular method for AGV navigation. A rotating laser is mounted onto the vehicle. For navigation, multiple fixed reference points like reflective strips, are located in the operating area on known coordinates. The coordinates of the reflectors are added to the global map. The emitted laser beams are reflected and scanned by the AGV after which the $\mathrm{AGV}$ is able to triangulate its absolute position based on the coordinates of the reflectors. At least three landmarks have to be visible to be able to navigate. This is a very accurate, secure and reliable method and is now used as a standard in a lot of AGV-systems. Disadvantages are the high price and the effort to place all the reflectors in the factory area.

3) GPS navigation: In GPS navigation [55], satellites with known positions into the global map emit signals which are detected by the GPS-receiver. This receiver can then measure the distance to each satellite. This info is used to determine the absolute position of the receiver using trilateration. At least four satellites have to be visible to be able to navigate. For this technique, a clear line of sight to the sky is needed. This is difficult to obtain in industrial environments. As an alternative, a Local Positioning Radar (LPR) in the factory can be used instead of satellites. The disadvantage of this LPR is that there is a precision of $\pm 10 \mathrm{~cm}$, which is not very accurate. This can be improved using sensor fusion, see Section IV-C

4) Natural or contour navigation: This type of navigation uses a laser to scan the whole environment around the vehicle [55]. Also, a Light Detection And Ranging (LiDAR) sensor can be used. No fixed landmarks like reflectors are needed. The AGV uses the existing environment to navigate. This makes this type of navigation very flexible. Unfortunately, the method is not that precise and robust. Using the scanned map of the environment, the vehicle is able to make a $2 \mathrm{D}$ map of its surroundings with all visible features like walls and pillars. When comparing this local map with the whole map of the factory, the robot knows its position into the map. This is also the way people navigate in a known environment. Before this can be done, the robot needs a map of its environment which can be scanned by a person and built up using Simultaneous Localization And Mapping (SLAM) [59]. Using SLAM, the robot has to explore the whole area while making local 2D-maps. These maps can then be combined into one large map of everything the robot has already seen. As a consequence, the system becomes a lot more flexible, especially in dynamic environments. The map can be updated every time there is a change in the environment. The disadvantages are that the material for this is expensive and that some transparent materials cannot be detected. Other sensors like sonar sensors can be used as an alternative.
5) Vision guided navigation: Vision-guided navigation is similar to contour navigation. Instead of using a LiDAR, a stereo camera is used to make images from which 3D-pointclouds can be built. Each pixel of the camera is converted to a point in the 3D-space which is situated before the camera. This $3 \mathrm{D}$-point-cloud consists of points which represent features in the area seen by the camera. This point-cloud can be projected onto a 2D-point-cloud which is a $2 \mathrm{D}$ projection of these features. An occupancy grid system [60] can be used to represent the local map of the environment. An occupancy grid is a cell decomposed representation of the environment, see Section V-B1. The whole area is divided into a grid of small squares. Each square is denoted either as "Unvisited", "Occupied" or as "Unoccupied". Also, a probability of occupation can be used. By projecting the 2D-point-cloud of the already seen features onto the occupancy grid, the features seen by the camera are translated into occupied cells. In this way, a map of the environment can be constructed by moving around and continuously projecting the seen $2 \mathrm{D}$-point-cloud onto the occupancy grid. Like in natural navigation, the robot also needs an initial map of its environment which can be scanned by a person when exploring the total area using SLAM. A disadvantage of this method is that camera images are light sensitive.

\section{Sensor fusion}

In practice, the above navigation methods are not implemented on their own. Because of noisy sensor data, the uncertainty on the measured position would be too large to properly navigate a vehicle. For this reason, these navigation methods are combined with filters or other types of navigation. This is called sensor fusion [61]. Sensor fusion can be divided into direct and indirect fusion. Direct fusion combines sensor data of different homogeneous or heterogeneous sensors. Indirect fusion combines sensor data with information of a prior knowledge of the environment and input.

- In direct fusion, different navigation techniques are combined. Two techniques which are not usable on their own because of high uncertainties are odometry and inertial navigation. These are frequently combined with other navigation techniques to obtain more accurate results. In odometry [55], the robot calculates its new position by knowing its starting position, the distance it already traveled, and the angle it is rotated. By using odometry sensors on the wheels, these distances and angles can be measured. However, this technique cannot be used as a stand-alone navigation technique as it is far from accurate because of slip of the wheels on different surfaces and other uncertainties. Because of this, odometry is used to combine with other navigation techniques to gain more accurate measurements. Inertial navigation [53] adds a gyroscope which detects and corrects the smallest change in the heading of the vehicle. Combination of inertial navigation and other navigation techniques will improve accuracy. 
- An example of an indirect technique is a combination of a navigation technique with some form of a Kalman Filter [62], [63]. This filter uses a model of the process to make a prediction of a next state using the current state and the properties of the process. This prediction is combined with the sensor measurement of this next state to obtain a more accurate estimation. The filter considers noise on sensor measurement and on the transition from one state to the next. The Kalman filter is a very commonly used technique to improve accuracy in mobile navigation.

In practice, sensor fusion is always implemented to have an accurate position estimation of the robot. Without this, the measurements would be too noisy to properly navigate a vehicle.

\section{Conclusion}

During the years, a variety of navigation techniques are developed. It is clear that flexible systems which are required for the future cannot work with physical circuits which are difficult to change, except for small aisles. AGVs need to be flexible and thus not fixed onto physical paths. For this reason, natural and vision-guided navigation methods will gain a lot more attention in the future. The disadvantage is that these methods are not very accurate and can be disturbed by a lot of factors like ambient light, vibrations, and uncertainties in measurements. Because of this, a combination of these techniques with the very accurate and robust laser navigation will be, in the opinion of the author, the future of AGV navigation. Currently, laser navigation is already the standard in offered AGV-systems. But when more new methods are added, the AGV can perceive a lot more of its surroundings and can keep a dynamic map of its environment including moving objects. Also, object recognition can be added in the future. This to detect hazardous situations, persons, or misplaced objects.

\section{Path Planning}

A next core AGV task is path planning [64], [65]. This can be seen as the static planning task of an AGV whereas motion planning, which is going to be handled in the next section, can be seen as dynamic path planning. Static planning means that a basic collision-free path is computed using already known information. No dynamic time-dependent elements are considered, only the already known map with obstacles is used. Using this map, the robot knows its free configuration space and the obstacle space. Path planning can thus be defined as the generation of an obstacle-free path, connecting the start point with the goal point, taking into account the geometric characteristics of obstacles and the kinematic constraints of the robot. Path planning consists of two steps:

- Representation of the free configuration space.

- Using a graph search algorithm to search for the shortest path using this representation

Although path planning is used to generate shortest paths, it is also frequently used in AGV-systems to calculate the cost to reach a certain goal. This information is frequently used in the task allocation algorithms. In this section, several methods to represent the environment and some search algorithms to compute the shortest path using this representation will be illustrated.

\section{A. Desired properties}

The goal of path planning is to generate the shortest path which minimizes an objective function. This objective can be travel time, travel distance or fuel consumption. Algorithms thus must be able to find a solution which connects the starting point and goal by minimizing this objection function. Path planning algorithms also have to be complete. An algorithm is said to be complete if it finds a solution or correctly reports that there is no solution, and this in a finite time. Incomplete planners, on the other hand, does not always find a solution when one exists. Another important property is time complexity. Path planning will be calculated a lot of times and also re-planning of paths will occur frequently. For this reason, the time complexity has to be as small as possible.

\section{B. Representation of the environment}

A path planning algorithm has as purpose to compute the shortest path. To do this, the algorithm first needs a representation of the possible reachable states of the AGV on the environmental map. In the current deployed AGV systems, the paths where these AGVs can move on are predetermined. Thus a circuit which consists of nodes (intersections) and segments (paths between the intersections) is defined and designed in, for example, graphical software. This network of nodes and segments is then used by a search algorithm to search for the shortest path from point A to B.

If there are no predefined paths available which the robot can follow, we need an algorithm to represent the configuration space in such a way that possible paths are generated into the full free configuration space. There are several algorithms for this. These algorithms are unfolded next.

1) Cell decomposition methods: In cell decomposition methods [66], the total environmental map is decomposed into a grid of cells with a certain size. Each cell is either defined as occupied or non-occupied. We call this also an occupancy grid. All the cells which are marked as occupied represent obstacles like walls, tables, or other structures. In the cells which are unoccupied, the robot is able to move. The occupancy can also be probabilistic. If a robot defines a cell multiple times as occupied because of what it perceives, the probability that the cell is occupied in reality is larger. Using this technique we can get a representation of the total area, knowing in which cells we can move (free configuration space) and in which cells not (obstacle space). This cell-based representation can be translated into a connectivity graph which represents the adjacency relations between cells. A graph search algorithm like an A*-algorithm can then be used to find an optimal path between the cell where the robot is situated and the goal cell. There are some different types of cell decomposition methods: 
a) Approximate decomposition: The size of all the cells is predefined and fixed. A disadvantage is that the complexity grows with the dimensions of the grid. And an obstacle which is smaller then the size of a cell will occupy the whole cell.

b) Adaptive cell decomposition: A large cell size is chosen at the beginning. If a cell is partially occupied by an obstacle, the cell is divided into four equal parts. This is repeated until each cell is either fully occupied or nonoccupied. This approach uses less memory but can result in difficulties in dynamic environments where a robot constantly needs to update its map when seeing other obstacles.

c) Exact cell decomposition: The map is decomposed into cells which are based on the map and the locations and shapes of the obstacles. No fixed size of the cells is predefined. The cells take over the shapes of the obstacles so the union of all the free cells represents exactly the free configuration space.

2) Trajectory maps: In this approach, the total area is covered with generated paths where the robot can move on. The generation of such trajectory maps can be done in several ways. A disadvantage is that the generation of such paths is not dynamic as the whole map has to be reconstructed if something changes in the map. This is not the case in cell-based representation where only the occupancy grid has to be updated.

a) Visibility graph: In this approach [67], a graph of possible paths to move on is constructed by connecting all the vertices of the obstacles which are represented as polygons with straight lines. The shortest path can then be calculated by using a simple graph search algorithm. One thing to mention here is that if the robot will move on segments which are made by connecting obstacle vertices, the robot will definitely collide with those objects. This can be prevented by enlarging the obstacles with the size of the robot itself. In this way, the robot has a graph of all possible paths it can move on without the possibility to collide with an object. So if its known that the graph consists of non-colliding paths, the only task is to let a graph search algorithm find the shortest path on the graph from the start to the goal.

b) Voronoi diagram: A Voronoi diagram [68] consists of a graph of lines which are equidistant from the two nearest obstacles. This guarantees that the paths to move on are positioned as far as possible from every obstacle. Obstacles here are also represented as polygons. Once the graph is constructed, a start and end point are added and connected to the graph and a graph search method is used to find an optimal solution. The AGV can then move on the vertices of the 2D-Voronoi diagram.

c) Probabilistic Road Maps (PRM): A PRM [69] makes use of random samples in the configuration space of the robot. If these random samples are in the free space, then they are connected to neighboring samples if the line between the two samples is collision-free. This can be the k-nearest neighbors or neighbors within a certain distance. This process of connecting the samples is continued until the graph is dense enough. After the graph is made, start and goal configurations are added to the graph. A graph search algorithm can now be used to connect the start and the goal with the shortest path. When the number of sampled points reaches infinity, a non-optimal path will certainly be found when there exists one.

d) Rapidly exploring random trees (RRT): In this RRT [70] algorithm, a tree is randomly expanded from the start node. An edge is only added to the tree if it does not cause a collision. Using this technique, an area can be rapidly covered by a tree of paths where an AGV can move on. An algorithm where two trees grow towards each other can also be used. One starting from the start and one starting from the goal, this is called bi-directional search. This is used to fasten up the process. These two trees will then be linked together. If the start and goal node is connected to the tree, a graph search algorithm is used to find the shortest path. If the number of expanded nodes approach infinity, a non-optimal path will certainly be found when one exists.

3) Artificial potential fields: Using artificial potential fields [71], a robot finds a path where it can move on by a superposition of all potential fields the robot senses. There are repulsive fields emitted by obstacles with a force inversely proportional with the distance to the obstacle. There are also attractive forces which are emitted by goal states. By using this method, it is possible that the robot gets stuck into local minima or that is can find a non-optimal path.

\section{Graph search algorithms}

In the previous part, alternative representations of the environment are given. These representations are further used by an algorithm to compute the shortest path. Here we want to notice again that these generated representations are only used in situations where predefined paths are missing. But in all current AGV systems, these AGVs move on predefined circuits. These circuits are used as a base to do path planning. Some of the algorithms to do this planning are unfolded next.

1) $A^{*}$-algorithm: The $\mathrm{A}^{*}$-heuristic algorithm [72] is one of the most popular classical graph search algorithms in calculating the least-cost path on a weighted graph. This algorithm uses a weighted graph with nodes as locations and edges between these nodes containing the cost to go from one node to another. Also, a list of unvisited nodes and a list of visited nodes are maintained. The algorithm makes use of a heuristic to find an optimal solution much faster, this can be an estimated cost from a node to the goal node. The algorithm starts from the initial start node and works towards the goal by visiting and evaluating each neighboring node in the unvisited list. It is an efficient and complete algorithm but has high memory usage. $A^{*}$ guarantees to find the optimal shortest path if there is one. 
2) $D^{*}$ Lite-algorithm: $\mathrm{D}^{*}$ Lite is an extension of $\mathrm{A}^{*}$. In contrast with $\mathrm{A}^{*}, \mathrm{D}^{*}$ Lite [72] works in the opposite direction which is from the goal to the start. Also different is the use of an extra parameter rhs which is introduced to give info about the cost of one-step ahead. Also, a heuristic is used here but one which estimates a cost to the start. This algorithm is an incremental heuristic search algorithm which re-uses trees from previous searches. This to speed up the search process. The $\mathrm{D}^{*}$ Lite-algorithm has good results in large and complex areas. It plans shorter paths much faster then $\mathrm{A}^{*}$-algorithms. It is less effective than $\mathrm{A}^{*}$ in simple and small areas. Whereas $A^{*}$ cannot do re-planning, $D^{*}$ Lite can re-plan because it keeps previous information. Because of this, $\mathrm{D}^{*}$ Lite algorithm is the most widely used path planning algorithm.

3) Other algorithms: The previously mentioned algorithms are the most common in path planning for AGVs. But there are more general optimization algorithms which can be used. Some which are already mentioned in the section about task allocation like Tabu Search, Genetic Algorithm, Particle Swarm Optimization, Ant colony algorithms, and Simulated Annealing can be used. But however, the graph search algorithms like $\mathrm{A}^{*}$ and $\mathrm{D}^{*}$ Lite, are the most common for mobile robotics.

\section{Conclusion}

There are several methods to represent environments and several algorithms to solve shortest path problems. Cell-based and generated trajectory map approaches are more used for environments when there are no predefined paths and where a robot can move freely into the free configuration space. But in current deployed AGV systems which work centralized, the representation of the environment is predetermined and graph-based (a collection of nodes and edges). Using this graph-based circuits, a graph search algorithm is used to find the shortest path from start to goal. In the future, due to decentralization, more flexible methods will be used. But however, the system still has to be predictable. If AGVs can move freely in the factory without following any predefined circuits, then the behavior of the AGV will not be predictable anymore for employees. So in the opinion of the author, these predefined graphical-designed graph-based circuits will still be used as a base in future systems together with the more dynamic path planning types. So the AGV can mainly move on predefined fixed paths but can leave the path and move freely when, for example, something is blocking the path. The same graph searching algorithms to find the shortest paths and to find costs to reach a given goal will be used as they have proven their utility in current systems.

\section{MOTION Planning}

In Section V, the static path planning is described. A basic path is constructed only considering the basic static obstacles like walls and racks. But in reality, this static path is not collision-free. During the execution of the path, the AGV can face obstacles where the static path planner does not know about. These obstacles can be unforeseen static obstacles, people, and other moving AGVs. Obviously, also collision with these features has to be prevented. Next to collisions, also deadlocks [73] need to be prevented. A deadlock is a situation where an AGV has no possible actions anymore. It cannot move forwards either backward.

The modification of the static predetermined path to avoid collisions and deadlocks is called motion planning [64]. The authors divide this motion planning into three subtasks:

- Collision Avoidance

- Deadlock Avoidance

- Zone Control

Zone control is the management of a particular zone to prevent a limited amount of AGVs to be exceeded into this zone. This is actually also a precaution to avoid deadlocks and collisions or to avoid too busy areas in general.

Motion planning can generally be divided into centralized and decentralized motion planning. This based on the information they use. Centralized planners make use of global information and decentral planners use local information. These are described in the next sections.

\section{A. Centralized motion planning}

Centralized motion planning is currently used in most of the industrial AGV systems. Collision-free trajectories are planned for all the robots simultaneously. Central motion planning makes use of global information. The central unit knows all the AGV positions, their goals, and the static paths they are about to execute. An optimization process uses all this information to search a solution space for an optimal solution. Although this central optimization approach is very powerful as it can consider this global information, it is constrained by the computational time requested for a real-time motion of the agents, which increases with the number of used AGVs. By considering this large amount of information, the optimization algorithm asks a lot of memory and computation time. This grows with the number of AGVs in the system which is therefore limited. Also the central unit acts as a single point of failure. A more flexible, scalable, and robust approach will be discussed in the next section.

\section{B. Decentralized motion planning}

In decentralized approaches, the motion planning is done in a distributed way. After a fixed predefined path is calculated minimizing a certain cost, the AGVs use their local information and perception to react upon unforeseen circumstances while executing this path. This is exactly how humans control their movement. We can only consider what we see around us and act upon that to avoid collisions or deadlocks. The coordination is performed by each AGV and its neighbors that share a coordination protocol. This way of motion coordination is much more robust and scalable as an AGV locally computes a plan using local information. In this way, the information and intelligence are distributed which eliminates the single point of failure and the limit of $\mathrm{AGVs}$ which can be added to the system. In the following section, different central and decentralized motion planning techniques and algorithms will be unfold. 


\section{Collision avoidance}

In collision avoidance, collisions with static or dynamic objects which cross the trajectory of an AGV are avoided. The most simple way to do this is to use a safety scanner which keeps up a safety zone. When objects come into this safety zone, the AGV slows down and finally stops. After the path is cleared, the AGV continues its path. More advanced methods can be used which try to move around an object or plan an alternative route. In the next sections, we cover central collision avoidance algorithms as well as decentral algorithms.

1) Centralized collision avoidance: To avoid collisions in the system, the central computer uses global information to do an optimization. Using all the available information like positions, goals, and presumed paths for each $\mathrm{AGV}$, an optimizer can search the solution space looking for an optimal solution. A solution here is a collection of paths generated for each AGV to get to their goal. Several possible solutions will be generated until a non-collision solution is found. When a solution is generated, and thus paths for each $\mathrm{AGV}$ are calculated, time intervals for each segment can be determined. For each segment on the circuit, there can be tracked in which time intervals the segment is occupied by a certain AGV [26], [74]. If in a solution, a segment is occupied by two AGVs in the same time interval, this solution will be discarded. To come to a feasible solution, another path can be searched for one of the AGVs, or one of the vehicles can be slowed down [75]. The optimizer searches until an optimal collision-free configuration is obtained. In this way, collisions with other AGVs are prevented. This process can be repeated real-time to adapt to changes in the system. The central system continuously takes a snapshot of the current situation (positions and paths for each AGV) and tries to optimize this configuration online. This central collision avoidance optimization process can also be included in the task allocation optimization [26], [75], [76]. For a certain task-robot pair, the output of the motion planner can, for example, be used to calculate the total traveled cost of all the AGVs. This can then be compared with another task-robot pair in the search for the optimal solution. To conclude, a central collision avoidance algorithm uses one large optimization process considering the global information and generates a set of collision-free paths for each AGV as an output.

2) Decentralized collision avoidance: In decentralized collision avoidance, the AGV reacts upon what it perceives to avoid collisions. If it detects a possible collision on the way, it can decide to slow down and to stop until the path is cleared. Or it can try to move around the obstacle locally. Literature is full of different techniques which are described next.

a) Forward sensing: In centralized motion planners, the central system can only consider the movement of other AGVs. But this has no knowledge of moving persons or unforeseen objects which can block a path. For this reason, current industrial AGVs keep up a certain safety zone using an onboard safety sensor to react to such unforeseen circumstances. When an object or person comes into this zone, the AGV will first decrease speed and eventually comes to a stop. When the object or person is removed out of this safety zone, the AGV resumes its movement. In this way, current centralized AGVs also have some local motion planning in addition.

b) Re-planning using $A^{*}$ or $D^{*}$ : When the robot is executing its trajectory and an unforeseen object crosses the path, the calculated path can be re-planned [72]. The same algorithms for initial path planning can be used. But instead of the start position, the robot its current position is used. The same goal remains. If $\mathrm{A}^{*}$ is used, a totally new path has to be calculated as $A^{*}$ does not keep historical data. This new path is calculated using the map, the goal position, and the new start position. As computing this new path from scratch takes a long time, using $A^{*}$ is not very flexible. A better alternative for re-planning is the $\mathrm{D}^{*}$ Lite algorithm. This algorithm does keep historical data in its memory. The re-planning can thus be done much faster and efficient which makes this algorithm much more flexible and convenient for the job.

c) Local deviation from the path: This technique [77] uses local data to calculate a local deviation from the circuit. It consists of four steps: a safety check, leaving the roadmap, overtaking the obstacle, and return to the road map. If an object is detected during the safety check, the AGV builds a new segment which leaves the roadmap using a lane-change maneuver curve. This curve is a polynomial function and is obtained considering maximum curvature, steering-rate, a minimal deviation from the map and a safe distance to the obstacle. Thus the kinematics and dynamics of the robot are considered. To overtake the object, a line parallel to the roadmap is maintained without decreasing the distance to the road map. To return, another lane-changing curve is calculated using the same technique as to leave the road map.

d) Virtual force fields (VFF): The Virtual Force Fields [78] method uses an occupancy grid representation as mentioned in Section V-B1. The occupied cells will repel the robot away with repellent forces which are dependent on the concentration of occupied cells. The force is inversely proportional to the square of the distance between the robot and the cell. The robot will move along the gradient of the total field constructed by the superposition of all the repellent force fields. Disadvantages of this method are the difficulty with obstacles which are to close to each other, because of the repellent forces on both sides, the robot will not move in between. Even though there is a place for the robot to move in between. This method does not deal with kinematic and dynamic constraints.

e) Vector field histogram (VFH): The VFH-method [79] solves the problem in the previous section where the robot does not move between obstacles which are to close to each other. This approach also uses a 2D-histogram grid to represent the environment. But here, the cartesian histogram 
grid is reduced to a $2 \mathrm{D}$ polar histogram which is built around the position of the robot at the time. This histogram shows the obstacle density seen from the robot perspective. In each possible heading direction, an obstacle density is calculated. The direction of the robot is then chosen based on the least concentration of obstacles. This algorithm also does not deal with kinematic and dynamic constraints. For this reason, an alternative algorithm ( $\mathrm{VFH}+)[80]$ is proposed which employs a threshold hysteresis to improve the shape of the trajectory. A cost function is used to choose the best direction in a space of possible directions provided by the polar histogram. This method also considers the vehicle width by enlarging the cells containing obstacles.

f) Dynamic windows approach: This algorithm [81] also deals with dynamic and kinematic constraints. The algorithm makes use of a solution space which consists of all possible velocity vectors including every possible speed and every possible heading direction of the robot. The velocity vectors are actually arcs defined by a velocity vector $(v, w)$, where $v$ is a linear velocity and $w$ an angular velocity. This solution space is reduced with velocity arcs which are not physically reachable by the AGV. This for example due to kinematic or dynamic constraints. Also velocity arc vectors which cause collisions are removed. What remains is a solution space containing all velocity arc vectors which are reachable by the AGV and which do not tend to a collision. These arcs can be used to construct a path around an obstacle. Once this solution space is defined, an optimization algorithm can create a path using this set of arcs while optimizing a certain objective. A set of velocity arc vectors to be sequentially followed by the AGV is obtained which will avoid a possible collision.

g) Optimal Reciprocal Collision Avoidance (ORCA): ORCA [82] is a collision avoidance algorithm where each robot knows its own position and velocity, and the other robots their position and velocity range. Each robot calculates a velocity space of velocities which will definitely cause collision within a certain time $\tau$. The geometry of this space is constructed using the geometry details of the robots (for example radius), the position of the robots, and the $\tau$-constant. Using all the velocities which are not in this space of velocities which cause a collision, it will be certain that the robot does not collide for at least the period of time $\tau$. The robot creates a boundary between allowable and non-allowable velocity vectors to prevent choosing a vector which will cause a collision. From the remaining velocity space, the velocity is chosen which is the closest to a preferred velocity stated by the motion planner. This algorithm can be extended to more robots. By linearizing the constraints, this problem of searching for an optimal speed can be done using linear programming. Every time step, the robot senses the positions and velocities of other robots, compute its boundary space, selects a new velocity outside this boundary using linear programming, and applies the velocity to its actuators. h) Predictive models: This approach mimics human behavior. Imagine being in a crowded metro station with hundreds of people having their own goal in mind and searching for the most optimal path through this bunch of people. Although this is a very complex situation, almost none collisions happen. But what actually are the steps we take to fulfill this task? Every time step we perceive everyone around us and try to predict where they are going. This is possible as the probability that someone makes an abrupt movement is very rare. And dependent on all these predicted velocity directions of all those people, we are going to adapt our path and thus our own velocity direction or vector. An example of a predictive model is a model for collision avoidance which uses deep reinforcement learning [83]. By perceiving the locations of the surrounding moving elements time step by time step, a reinforcement learning algorithm is going to predict the appropriate action. This action can be chosen from an action space, which consists of velocity vectors from speed zero to the maximum speed and this in all directions. The robot has a value function which gives as output this velocity vector given the sequence of perceptions as input, and this step by step. This value function is learned by performing random actions and looking at the results of those actions. The robot is rewarded when reaching its goal but punished when coming too close to an obstacle. The coefficients of this value function are learned by a deep learning network which is trained using data retrieved from the previously described ORCA method. A large number of trajectories generated by this ORCA algorithm are used to train the network using deep learning.

\section{Deadlock Avoidance}

In literature, there are several ways to prevent deadlocks. First, the layout of the circuit where an AGV can move on can be designed in order to reduce deadlocks. This option is more related to the design of an AGV system. As we only focus on the control of AGVs, we will not cover this part as we are not focused on design. Second, the environment can be divided into several control zones in which the maximum amount of AGVs can be kept. This is also used to avoid collisions and is covered in the next section VI-E And thirdly, some routing strategies can be developed to prevent deadlocks. These are algorithms which inherit traffic rules or consensus between vehicles to avoid deadlocks. This is covered in the following sections. We make a distinction between central and decentral deadlock avoidance. Algorithms for both approaches are described next.

1) Central deadlock avoidance: Central deadlock avoidance is considered into the large optimization process together with collision avoidance as mentioned in Section VI-C1. The way this central deadlock avoidance can be included in the optimization can be done in different ways. Petri nets [84] are a frequently used approach for deadlock avoidance in central motion planning. A Petri net, or a place/transition net, is a graphical tool to represent systems with concurrency. It is a directed bipartite graph of nodes, transitions, and arcs. A node can be a condition or a state 
of the system, and a transition can be an event that may occur. The directed arcs describe the relationship between nodes and transitions. It clarifies which nodes are pre- and/or postconditions for which transitions. These nets can be used to model the AGV system layout and the paths that the vehicles follow [85]. Li et al. [86] give a review on the deadlock avoidance in automated manufacturing using Petri nets. Other algorithms use a strategy to detect a cyclic-waiting situation [87], using a graph theory [88], or using a matrix-based deadlock detection algorithm [89].

2) Decentral deadlock avoidance: In decentralized deadlock avoidance, AGVs try to avoid deadlocks using their local information. So only what they can observe, can be used to avoid deadlocks. This in contrast with centralized optimizers where the central unit has access to all the positions and future planned segments for each AGV. Markus et al. [90] proposes such a distributed algorithm which consist of a collision avoidance algorithm, a deadlock detection algorithm, and a deadlock resolution algorithm. When the distance between two robots drops below a certain value, the robots initiate a coordination link. One of the two robots is the coordinator and one is the partner. This link is used to detect and resolve deadlocks and to avoid collisions. The link either gives permission to the robot to move on or not. A similar approach [91] makes use of a two-layer architecture for path planning where the coordination among AGVs is based on a negotiation of shared resources. For each section on the static path, the AGV checks whether the section is available or occupied by another AGV. If more AGVs want the same segment, then an auction process is initiated. The winner can move on the segment. In [92] presumed collision-free trajectories are planned for each $\mathrm{AGV}$ by a central computer like in section VI-C1 These presumed paths are exchanged with neighboring AGVs so they know each AGV's intentions. Each AGV then uses the presumed trajectories of neighbors to locally compute a collision-free trajectory according to a priority policy. Dario et al. [93] proposes another decentralized deadlock avoidance algorithm based on a shared resources protocol and a replanning strategy. The coordination is based on a set of rules like in urban situations. The rules are used to get access to local resources. Another algorithm which makes use of traffic rules to coordinate robots is [94]. Basic rules like keeping sufficient space in front of the robot are used. In [95], another decentral deadlock avoidance algorithm which combines AI techniques with real-time control is presented.

\section{E. Zone Control}

Zone control is used in centralized as well as in decentralized approaches. In centralized systems, the maximum amount of AGVs allowed in a zone can be given to the optimizer as a constraint. It is one of the most effective strategies to prevent collisions with other AGVs. The use of zone-control eases the avoidance of collisions and deadlocks. This technique divides the circuit where the AGVs can move on into non-overlapping zones in which the maximum amount of vehicles is limited. Vehicles which want to enter a full zone have to wait or replan their route. Traditional techniques are fixed zone strategies where the zone areas are fixed. More new techniques make use of dynamic zone control techniques in which the size and arrangement of zones can vary. Li et al. [96] presents a traffic control scheme based on a novel discrete-event zone-control model. In [97], some zone design methods are covered and a dynamic zone strategy is proposed. In [98], another dynamic zone control is proposed. The objective here is to maintain the same workload for each vehicle. Zones are redesigned during the operation to avoid differences in workload between the zones. Reveliotis et al. [99] proposes a strategy that incrementally determines $\mathrm{AGV}$ routes one zone at a time. Fanti et al. [100] proposes another zone-based control where the guide paths are subdivided into disjoint zones representing intersections, straight lines or workstations. Only one vehicle can occupy a zone at a time and the permission to enter a zone must be given by the control.

\section{F. Conclusion}

Next, to task allocation, motion planning is another very complex core AGV task. It covers collision avoidance, deadlock avoidance, and zone control. Centralized approaches use global information for an optimization process to do the motion planning. Because they have access to all the information, they can prevent collisions and deadlocks by considering the whole movement of the fleet. They take a snapshot of the current AGV configuration and continuously optimize the allocation with respect to the current situation. This approach is widely used in today's industrial AGV systems. However, this approach inherits no flexibility and is very time-consuming. More flexible is when an AGV can react from the moment it encounters a problem. This is exactly how humans prevent collisions and deadlocks. By perceiving the local environment, we can act properly to changes at the moment. This is what decentralized motion planners do. They perceive their local surroundings and act upon them. The authors think that because of these properties, decentralized motion planners will be used more frequently in future industrial systems as these will become larger and more complex.

\section{Vehicle Management}

Vehicle management is the simplest core AGV task in that sense that it does not require complex algorithms or techniques. It monitors all the system parameters like battery status, error statuses, and mechanical parameters. All these parameters have to be monitored by a central unit or by the AGV itself while communicating its parameters to other AGVs. These parameters can cause constraints at the task allocation level. When a battery of an AGV is low or it finds itself in an error status, the tasks it was assigned to cannot be completed anymore. We can divide this management of vehicle statuses into a centralized or a decentralized structure:

- When a centralized approach is used, the central controller takes all these parameters into account. If it knows that the battery life of a vehicle is not enough to execute a certain task, then it will give it another 
less energy-asking task or it will send the AGV to the charging station. When the central unit receives error statuses form an AGV, then the central unit will not involve the $\mathrm{AGV}$ into the task allocation optimization anymore. Instead, it will send the AGV to maintenance.

- If decentralized approaches are used, the AGV monitors its own parameters. If it sees that its battery capacity is low, it will, in a market-based approach for instance, not bid on an energy expensive task but will directly head to a charging station. An AGV can also send its status parameters to other neighboring AGVs so they can maybe take over some tasks when the initial AGV has an error status, needs battery charging, or when it needs maintenance. The AVG deals independently with its own parameters.

This core task can be seen as an extra input to the other core AGV tasks. So when more decentralized task allocation and motion planning algorithms will be used in the future, also a decentralized management of the AGV's system parameters will be used. As the distribution of intelligence and information benefits future requirements, the authors believe that this decentralization of status management will grow in the future.

\section{CONCLUSIONS}

In this paper we covered the current and future-potential state-of-the-art techniques and algorithms to control an AGV system for a central and a decentral architecture. We decomposed the AGV control function into a set of core tasks and reviewed for each of those core tasks the available algorithms. In the commercially available offer, we see that current systems almost all work fully centralized but also discern a clear interest in decentralization as an element to meet needs like flexibility, openness, scalability, and robustness. The industry 4.0 paradigm will push AGV manufacturers to think about decentralization as an essential element to deal with these requirements as systems will become ever larger and complex. The authors think that more decentralized and market-based techniques are suited to introduce flexibility and scalability in the system. The abundance of literature teaches us that already a lot of algorithms exist to that effect in lab environments. Further work will focus on the practical implementation of these algorithms and combinations of these algorithms to real industrial environments.

\section{ACKNOWLEDGMENTS}

This work is supported by the M-group, part of the KU Leuven campus in Bruges.

\section{REFERENCES}

[1] J. Szpytko, "Automated Guided Vehicles Navigating Problem In Container Terminal", Logistics and Transport, vol. 2, no. 13, pp. 107-116, 2011.

[2] Grand View Research, "Automated Guided Vehicles Market”, 2018.
[3] T. Le-Anh and M. B. De Koster, "A review of design and control of automated guided vehicle systems", European Journal of Operational Research, vol. 171, no. 1, pp. 1-23, 2006.

[4] I. F. Vis, "Survey of research in the design and control of automated guided vehicle systems", European Journal of Operational Research, vol. 170, no. 3, pp. 677-709, 2006.

[5] X. Jia and M. Q. Meng, "A survey and analysis of task allocation algorithms in multi-robot systems", IEEE International Conference on Robotics and Biomimetics, ROBIO 2013, pp. 2280-2285, 2013.

[6] N. Kokash, "An introduction to heuristic algorithms", 2018.

[7] S. Nesmachnow, "An overview of metaheuristics: accurate and efficient methods for optimisation", International Journal of Metaheuristics, vol. 3, no. 4, pp. 320-347, 2014.

[8] A. R. Mosteo and L. Montano, "A survey of multirobot task allocation", 2010, pp. 1-27.

[9] B. P. Gerkey and M. J. Mataric, "A formal analysis and taxonomy of task allocation in multi-robot systems", International Journal of Robotics Research, vol. 23, no. 9, pp. 939-954, 2004.

[10] T. Vidal and J. Bidot, "Dynamic sequencing of tasks in simple temporal networks with uncertainty", $C P 2001$ Workshop in Constraints and Uncertainty, pp. 1-10, 2001.

[11] K. Erol, J. Hendler, and D. S. Nau, "HTN planning: Complexity and expressivity", in In Proceedings of the Twelfth National Conference on Artificial Intelligence, AAAI Press, 1994, pp. 1123-1123.

[12] A. Khamis, A. Hussein, and A. Elmogy, "Multi-Robot Task Allocation: A Review of the State-of-the-Art", Cooperative Robots and Sensor Networks, vol. 2, pp. 31-51, 2015.

[13] N. Atay and B. Bayazit, "Mixed-Integer Linear Programming Solution to Multi-Robot Task Allocation Problem", Tech. Rep. 314, 2006.

[14] R. A. Abdeen, "An Algorithm for String Searching Based on Brute-Force Algorithm", Journal of Computer Science and Network Security, vol. 11, no. 7, pp. 24-27, 2011.

[15] C. S. Edwards, "A branch and bound algorithm for the generalized assignment problem", Mathematical Programming, vol. 8, pp. 91-103, 1975.

[16] B. Coltin and M. Veloso, "Mobile Robot Task Allocation in Hybrid Wireless Sensor Networks", International Conference on Intelligent Robots and Systems, pp. 2932-2937, 2010.

[17] S. Giordani, M. Lujak, and F. Martinelli, A distributed algorithm for the multi-robot task allocation problem, 2010.

[18] W. Kmiecik, M. Wojcikowski, L. Koszalka, and A. Kasprzak, "Task allocation in mesh connected processors with local search meta-heuristic algorithms", Lecture Notes in Computer Science, pp. 215-224, 2010. 
[19] W. Zang, "NSA algorithm and its computational complexity-preliminary results", IEEE Transactions on Applications and Industry, pp. 442-446, 1989.

[20] E. K. Burke and Y. Bykov, "A late acceptance strategy in hill-climbing for exam timetabling problems", in Proceedings of the Conference on the Practice and Theory of Automated Timetabling (PATAT 2008), 2008, pp. 1-7.

[21] A. R. Mosteo and L. Montano, "Simulated annealing for multi-robot hierarchical task allocation with flexible constraints and objective functions", IROS'06 workshop on Network Robot Systems: Toward intelligent robotic systems integrated with environments, pp. 1-8, 2006.

[22] M. D. Besten, T. Stutzle, and M. Dorigo, "Design of iterated local search algorithms An Example application to the single machine total weighted tardiness problem", Lecture Notes in Computer Science (including subseries Lecture Notes in Artificial Intelligence and Lecture Notes in Bioinformatics), pp. 441-451, 2001, ISSN: 16113349. DOI: 10.1007/3-540-45365-2_46

[23] P. Hansen and N. Mladenović, "A Tutorial on Variable Neighborhood Search", Les Cahiers du GERAD, 2003.

[24] W. H. Chen and C. S. Lin, "A hybrid heuristic to solve a task allocation problem", Computers and Operations Research, vol. 27, no. 3, pp. 287-303, 2000.

[25] P. J. Shea, K. Alexander, and J. Peterson, "Group tracking using genetic algorithms", Proceedings of the 6th International Conference on Information Fusion, FUSION 2003, vol. 1, pp. 680-687, 2003.

[26] C. Liu and A. Kroll, A centralized multi-robot task allocation for industrial plant inspection by using $A^{*}$ and genetic algorithms, 2012.

[27] R. Poli, J. Kennedy, and T. Blackwell, "Particle swarm optimization", Swarm Intelligence, vol. 1, pp. 33-57, 2007.

[28] P. Moscato, U. E. D. Campinas, C. Cotta, and C. D. Teatinos, "A Gentle Introduction to Memetic Algorithms", in Handbook of Metaheuristics, F. Glover and G. Kochenberger, Eds., 1st ed., Kluwer Academic Publishers, 2003, ch. 10.1007/0-, pp. 1-36.

[29] D. Yingying, H. Yan, and J. Jingping, "Multi-Robot Cooperation Method Based On The Ant Algorithm", IEEE Swarm Intelligence Symposium, pp. 14-18, 2003.

[30] E. K. Burke, M. R. Hyde, G. Kendall, G. Ochoa, E. Özcan, and J. R. Woodward, "A classification of hyper-heuristic approaches", International Series in Operations Research and Management Science, vol. 272, pp. 453-477, 2010.

[31] A. Langevin, D. Lauzon, and D. Riopel, "Dispatching, routing, and scheduling of two automated guided vehicles in a flexible manufacturing system", International Journal of Flexible Manufacturing Systems, vol. 8, no. 3, pp. 247-262, 1996.

[32] R. G. Smith, "The Contract Net Protocol: High-Level Communication and Control in a Distributed Problem
Solver", IEEE Transactions on Computers, vol. C-29, no. 12 , pp. $1104-1113,1980$.

[33] A. Viguria, I. Maza, and A. Ollero, "SET: An algorithm for distributed multirobot task allocation with dynamic negotiation based on task subsets Antidio", IIEEE International Conference on Robotics and Automation, pp. 3339-3344, 2007.

[34] H. L. Choi, L. Brunet, and J. P. How, "Consensusbased decentralized auctions for robust task allocation”, IEEE Transactions on Robotics, vol. 25, no. 4, pp. 912-926, 2009.

[35] P. A. Gao, Z. X. Cai, and L. L. Yu, "Evolutionary computation approach to decentralized multi-robot task allocation", 5th International Conference on Natural Computation, ICNC 2009, vol. 5, pp. 415-419, 2009.

[36] S. Botelho and R. Alami, "M+: a scheme for multirobot cooperation through negotiated task allocation and achievement", Proceedings 1999 IEEE International Conference on Robotics and Automation, vol. 2, pp. 1234-1239, 1999.

[37] M. Lagoudakis, M. Berhault, S. Koenig, P. Keskinocak, and A. Kleywegt, "Simple auctions with performance guarantees for multi-robot task allocation", 2004 IEEE/RSJ International Conference on Intelligent Robots and Systems (IROS) (IEEE Cat. No.04CH37566), vol. 1, pp. 698-705, 2004.

[38] S Koenig, C Tovey, and M Lagoudakis, "The power of sequential single-item auctions for agent coordination", Proceedings of the AAAI Conference on Artificial Intelligence, pp. 1625-1629, 2006.

[39] E. Nunes and M. Gini, "Multi-Robot Auctions for Allocation of Tasks with Temporal Constraints", AAAI Conference on Artificial Intelligence, pp. 2110-2116, 2000.

[40] M. Mcintire, E. Nunes, and M. Gini, "Iterated MultiRobot Auctions for Precedence-Constrained Task Scheduling", in Proceedings of the 15th International Conference on Au- tonomous Agents and Multiagent Systems (AAMAS 2016), 2016, pp. 1078-1086.

[41] E. Nunes, M. McIntire, and M. Gini, "Decentralized multi robot allocation of tasks with temporal and precedence constraints", Advanced Robotics, vol. 31, no. 22, pp. 1193-1207, 2017.

[42] D.-H. Lee, "Resource-based task allocation for multirobot systems", Robotics and Autonomous Systems, vol. 103, pp. 151-161, 2018.

[43] C. Sung, N. Ayanian, and D. Rus, "Improving the performance of multi-robot systems by task switching", Proceedings - IEEE International Conference on Robotics and Automation, pp. 2999-3006, 2013.

[44] A. Viguria, I. Maza, and A. Ollero, "S+T: An algorithm for distributed multirobot task allocation based on services for improving robot cooperation", Proceedings - IEEE International Conference on Robotics and Automation, pp. 3163-3168, 2008.

[45] M. B. Dias, "TraderBots: A New Paradigm for Robust and Efficient Multirobot Coordination in Dynamic Environments", Tech. Rep., 2004. 
[46] B. P. Gerkey and M. J. Matarić, "Sold!: Auction methods for multirobot coordination", IEEE Transactions on Robotics and Automation, vol. 18, no. 5, pp. 758-768, 2002.

[47] D. Weyns, N. Bouck, K. Schelfthout, and T. Holvoet, "Dyncnet: A protocol for flexible task assignment applied in an AGV transportation system", CEUR Workshop Proceedings, vol. 223, no. January, 2006.

[48] L. E. Parker, "ALLIANCE: An architecture for fault tolerant multirobot cooperation", IEEE Transactions on Robotics and Automation, vol. 14, no. 2, pp. 220240, 1998.

[49] B. B. Werger and M. J. Mataric, "Broadcast of Local Eligibility for Multi-Target Observation", in Parker L.E., Bekey G., Barhen J. (eds) Distributed Autonomous Robotic Systems, vol. 4, Tokyo: Springer, 2000, pp. 347-356.

[50] T. Dahl, M. Mataric, and G. Sukhatme, "Multi-robot task-allocation through vacancy chains", IEEE International Conference on Robotics \& Automation, vol. 2, pp. 12-17, 2003

[51] D. Weyns, N. Boucké, and T. Holvoet, "Gradient field-based task assignment in an AGV transportation system", Proceedings of the fifth international joint conference on Autonomous agents and multiagent systems - AAMAS '06, 2006.

[52] G. J. Cawood and I. A. Gorlach, "Navigation and locomotion of a low-cost Automated Guided Cart", Proceedings of the 2015 Pattern Recognition Association of South Africa and Robotics and Mechatronics International Conference, PRASA-RobMech 2015, pp. 83-88, 2015.

[53] H. M. Barbera, J. P. C. Quinonero, M. A. Z. Izquierdo, and A. G. Skarmeta, "i-Fork: a flexible AGV system using topological and grid maps", in IEEE International Conference on Robotics \& Automation, IEEE, 2003, pp. 2147-2152.

[54] J. Song, "Electromagnetic Induction Sensor of Navigation System for Spraying Robot *", Proceedings of the 2011 International Conference on Informatics, Cybernetics, and Computer Engineering (ICCE2011), pp. 175-181, 2011.

[55] C. Feledy and M. S. Luttenberger, "A State of the Art Map of the AGVS Technology and a Guideline for How and Where to Use It", University of Lund, Lund, Tech. Rep., 2017.

[56] S. Kamewaka and S. Uemura, "A magnetic guidance method for automated guided vehicles", IEEE Transactions on Magnetics, vol. 23, no. 5, pp. 2416-2418, 1987.

[57] S. Y. Lee and H. W. Yang, "Navigation of automated guided vehicles using magnet spot guidance method", Robotics and Computer-Integrated Manufacturing, vol. 28, no. 3, pp. 425-436, 2012.

[58] U. Andersson, "Laser Navigation System for Automatic Guided Vehicles", Lulea, Tech. Rep., 2013.

[59] H. Durrant-whyte and T. Bailey, "Simultaneous Localisation and Mapping (SLAM): Part I The Essential
Algorithms", IEEE Robotics \& Automation Magazine, vol. 13, no. 2, pp. 99-110, 2006.

[60] A. M. Santana, K. R. Aires, R. M. Veras, and A. A. Medeiros, "An approach for 2D visual occupancy grid map using monocular vision", Electronic Notes in Theoretical Computer Science, vol. 281, pp. 175-191, 2011.

[61] W. Elmenreich, "An introduction to sensor fusion", Vienna University of Technology, Austria, pp. 1-28, 2002.

[62] G. Welch and G. Bishop, "An Introduction to the Kalman Filter", in Siggraph Course. 8, 2006, pp. 1-16.

[63] S. W. Yoon, S. B. Park, and J. S. Kim, "Kalman filter sensor fusion for Mecanum wheeled automated guided vehicle localization", Journal of Sensors, vol. 2015, pp. 1-8, 2015

[64] V. Kunchev, L. Jain, V. Ivancevic, and A. Finn, "Path Planning and Obstacle Avoidance for Autonomous Mobile Robots: A Review", in KES2006 10th International Conference on Knowledge-Based \& Intelligent Information \& Engineering Systems, 2006, pp. 537544.

[65] S. G. Anavatti, S. L. Francis, and M. Garratt, "Pathplanning modules for Autonomous Vehicles: Current status and challenges", ICAMIMIA 2015 - International Conference on Advanced Mechatronics, Intelligent Manufacture, and Industrial Automation, Proceeding - In conjunction with Industrial Mechatronics and Automation Exhibition, IMAE, pp. 205-214, 2016.

[66] Ahmad Abbadi and Vaclav Prenosil, "Safe Path Planning Using Cell Decomposition Approximation", International Conference Distance Learning, Simulation and Communication, no. May, 2015.

[67] J. O'Rourke and I. Streinu, "The vertex-edge visibility graph of a polygon", Computational Geometry: Theory and Applications, vol. 10, no. 2, pp. 105-120, 1998.

[68] Adam Dobrin, "A Review of Properties and Variations of Voronoi Diagrams", World Applied Sciences Journal, vol. 21, no. 1, pp. 21-29, 2013.

[69] L. E. Kavraki, P. Svestka, J.-C. Latombe, and Mark H. Overmars, "Probabilistic Roadmaps for Path Planning in High-Dimensional Configuration Spaces", IEEE Transactions on Robotics and Automation, vol. 12, no. 4, pp. 566-580, 1996.

[70] S. M. LaValle and J. J. Kuffner, "Rapidly-Exploring Random Trees: Progress and prospects", Algorithmic and computational robotics: New directions, 2000.

[71] C. Warren, Global path planning using artificial potential fields, 1989.

[72] D. H. Kim, N. T. Hai, and W. Y. Joe, "A Guide to Select Path Planning Algorithm for Automated Guided Vehicle (AGV)", Lecture Notes in Electrical Engineering, vol. 465, pp. 587-596, 2018.

[73] K. M.R. L. Moorthy and W. H. Guan, "Deadlock Prediction and Avoidance in an AGV System", Tech. Rep., 2000.

[74] S. C. Srivastava and A. K. Choudhary, "Development of an intelligent agent-based AGV controller 
for a flexible manufacturing system", International Journal Advanced Manufacturing Technology, vol. 36, pp. 780-797, 2008.

[75] D. K. Liu and A. K. Kulatunga, "Simultaneous planning and scheduling for multi-autonomous vehicles", Studies in Computational Intelligence, vol. 49, pp. 437-464, 2007.

[76] K. Jose and D. K. Pratihar, "Task allocation and collision-free path planning of centralized multi-robots system for industrial plant inspection using heuristic methods", Robotics and Autonomous Systems, vol. 80, pp. 34-42, 2016.

[77] V. Digani, F. Caramaschi, L. Sabattini, C. Secchi, and C. Fantuzzi, "Obstacle avoidance for industrial AGVs", Proceedings - 2014 IEEE 10th International Conference on Intelligent Computer Communication and Processing, ICCP 2014, pp. 227-232, 2014.

[78] Johann Borenstein and Yorem Koren, "Real-Time Obstacle Avoidance for Fast Mobile Robots", IEEE Transactions on Systems, Man, and Cybernetics - Part A: Systems and Humans, vol. 19, no. 5, pp. 1179-1187, 1989.

[79] J Borenstein and Y Koren, "The vector field histogram - fast obstacle avoidance for mobile robots", IEEE Journal of Robotics and Automation, vol. 7, no. 3, pp. 278-288, 1991.

[80] I. Ulrich and J. Borenstein, "VFH+: Reliable obstacle avoidance for fast mobile robots", Proceedings - IEEE International Conference on Robotics and Automation, vol. 2, pp. 1572-1577, 1998.

[81] D. Fox, W. Burgard, and S. Thrun, "The Dynamic Window Approach to Collision Avoidance", IEEE Robotics \& Automation Magazine, vol. 4, no. 1, pp. 23-33, 1997.

[82] S. J. Guy, M. Lin, and D. Manocha, "Reciprocal n -body Collision Avoidance", in Robotics Research, STAR 70, Berlin Heidelberg: Springer, 2011, pp. 1-16.

[83] Y. F. Chen, M. Liu, M. Everett, and J. P. How, "Decentralized non-communicating multiagent collision avoidance with deep reinforcement learning", Proceedings - IEEE International Conference on Robotics and Automation, pp. 285-292, 2017.

[84] W. Hu, Y. Zhu, and J. Lei, "The Detection and Prevention of Deadlock in Petri Nets", Physics Procedia, vol. 22, pp. 656-659, 2011.

[85] M. P. Fanti, "A deadlock avoidance strategy for AGV systems modelled by coloured Petri nets", Proceedings - 6th International Workshop on Discrete Event Systems, WODES 2002, pp. 61-66, 2002.

[86] Z. Li, N. Wu, and M. Zhou, "Deadlock control of automated manufacturing systems based on petri nets-a literature review", IEEE Transactions on Systems, Man and Cybernetics Part C: Applications and Reviews, vol. 42, no. 4, pp. 437-462, 2012.

[87] R. Lochana Moorthy, W. Hock-Guan, N. WingCheong, and T. Chung-Piaw, "Cyclic deadlock prediction and avoidance for zone-controlled AGV sys- tem", International Journal of Production Economics, vol. 83, no. 3, pp. 309-324, 2003.

[88] J. W. Yoo, E. S. Sim, C. Cao, and J. W. Park, "An algorithm for deadlock avoidance in an AGV System", International Journal of Advanced Manufacturing Technology, vol. 26, no. 5-6, pp. 659-668, 2005.

[89] M. Lehmann, M. Grunow, and H. O. Günther, "Deadlock handling for real-time control of AGVs at automated container terminals", Container Terminals and Cargo Systems: Design, Operations Management, and Logistics Control Issues, vol. 657, pp. 215-241, 2007.

[90] M. Jäger and B. Nebel, "Decentralized collision avoidance, deadlock detection, and deadlock resolution for multiple mobile robots", IEEE International Conference on Intelligent Robots and Systems, vol. 3, pp. 1213-1219, 2001.

[91] V Digani, L Sabattini, and C Secchi, "Towards decentralized coordination of multi robot systems in industrial environments: A hierarchical traffic control strategy", in IEEE 9th International Conference on Intelligent Computer Communication and Processing (ICCP), IEEE, 2013, pp. 209-215.

[92] G. Demesure, M. Defoort, A. Bekrar, D. Trentesaux, and M. Djemai, "Decentralized Motion Planning and Scheduling of AGVs in an FMS", IEEE Transactions on Industrial Informatics, vol. 14, no. 4, pp. 1744 1752, 2018.

[93] D. Marino, A. Fagiolini, and L. Pallottino, Distributed Collision-free Protocol for AGVs in Industrial Environments, 2011.

[94] S. Kato, S. Nishiyama, and J. Takeno, "Coordinating Mobile Robots by Applying Traffic Rules", IEEE/RSJ International Conference on Intelligent Robots and Systems, pp. 1535-1541, 1992.

[95] L. Chun, Z. Zheng, and W. Chang, “A Decentralized Approach to the Conflict-Free Motion Planning for Multiple Mobile Robots", in Proceedings of the 1999 IEEE International Conference on Robotics \& Automation, 1999.

[96] Q. Li, J. T. Udding, and A. Pogromsky, "Zone-ControlBased Traffic Control of Automated Guided Vehicles", Coordination Control of Distributed Systems, pp. 5360, 2015.

[97] Y. C. Ho and T. W. Liao, "Zone design and control for vehicle collision prevention and load balancing in a zone control AGV system", Computers and Industrial Engineering, vol. 56, no. 1, pp. 417-432, 2009.

[98] Y. C. Ho, "Dynamic-zone strategy for vehicle-collision prevention and load balancing in an AGV system with a single-loop guide path", Computers in Industry, vol. 42, no. 2, pp. 159-176, 2000.

[99] S. A. Reveliotis, "Conflict resolution in AGV systems", IIE Transactions (Institute of Industrial Engineers), vol. 32, no. 7, pp. 647-659, 2000.

[100] M. P. Fanti, "Event-based controller to avoid deadlock and collisions in zone-control AGVS", International Journal of Production Research, vol. 40, no. 6, pp. 1453-1478, 2002. 